\title{
Studying the Interrelationship amongst various Barriers to Implementing Corporate Social Responsibility in Indian Textile Industry
}

\author{
Bhoomica Aggarwal \\ HCL Technologies Private \\ Limited, Sector 125, Noida \\ India
}

\author{
Remica Aggarwal \\ School of Business, \\ University of Petroleum \& \\ Energy Studies, Dehradun
}

\author{
Veena Aggarwal \\ Recventures Education \\ Services Private Limited, \\ Delhi, India
}

\begin{abstract}
It is a world known fact that businesses cannot survive alone . They are required to deal with expectations from its various stakeholders . They therefore should follow the ethical principles in their engagements to maintain their corporate social responsibility. However implementation of CSR principles is not an easy task particularly in developing countries such as India . Furthermore, it also depends on the kind of industry. CSR is applicable to various industries throughout the globe, including manufacturing, automobiles, supply chains, pharmaceuticals, textiles, and so on. However, the underlying processes in the relationship between the degree of development of CSR in companies and the drivers/barriers that determine this development are still at the centre of an intense debate. This paper focuses on identifying various barriers for implementation of corporate social responsibility in Indian textile industry . Further it studies the interrelationship amongst them using ISM methodology.
\end{abstract}

\section{Keywords}

Corporate social responsibility, ISM methodology, Indian textile industry

\section{INTRODUCTION}

Businesses have evolved from the liberal models of 1970s to a stakeholder model of 1990s (KPMG , 2008). While the former stresses on the economic bottom line of business which advocates that business is for business, latter focuses on the growing realization that with profits, organizations also have societal roles to fulfil. This has been marked by the acceptance of the Triple bottom line concept - Business for, "people, planet and profit." It is widely accepted fact that every business require to satisfied its diverse stakeholders in order to survive. They therefore have to behave ethically and contribute to social and economic development while simultaneously improving the quality of life of the workforce and their families . As per National Voluntary guidelines, India (2011), "businesses have to endeavour to become responsible actors in society, so that their every action leads to sustainable growth and economic development. Accordingly, the Guidelines use the terms 'Responsible Business' instead of Corporate Social Responsibility (CSR)".

Various factors have led to an increased focus on the notion of corporate social responsibility. According to Aguinis, H., \& Glavas, A (2012), institutional and stakeholder pressures, increased regulatory environment, enhancement of business standards, changing imperatives of national and global governance, trends towards disclosure of corporate performance in the area of social and environmental performance apart from economic parameters, increased competitiveness as well as firm's own self of responsibility and justice are some of the factors which have contributed to bring the spotlight on Corporate Social Responsibility. Though CSR is applicable to various industries throughout the globe, including manufacturing, automobiles, supply chains, pharmaceuticals, textiles, and so on , it also faces lot of constraints and barriers towards its successful implementation particularly in developing countries such as India . Among industrial sectors, the textiles industry garners significant attention regarding its workability issues, which include the balance of workers' resources and their work demands (Dash 2009). Recently, the textiles industry has faced a huge crisis over CSR issues particularly in south Asian region due to various hidden factors. Hence, this study attempts to investigate the factors that resist the successful implementation of CSR in the Indian textile sector.

Paper is written as follows. Section 2 provides a review of existing literatures to identify prominent research already conducted on the barriers of CSR. Section 3 presents the ISM methodology. Section 4 presents the case problem. Section 5 presents the managerial implications.

\section{LITERATURE REVIEW ON CSR BARRIERS IN TEXTILE INDUSTRY}

Recently, many organizations have felt free to implement CSR in wider arenas, and Indian textile industries, in particular, have worked hard to enact CSR. Over the last two decades, India's textile sector has expanded from a largely domestic market to the global marketplace, and this broader business environment has required the adoption of modern workplace strategies. Those required business strategies include ethical and social responsibilities, keeping costs low, offering just-in-time delivery, and shortening lead times, etc. On the contrary in a study by Balasubramanian et al. (2005), various barriers to CSR implementation were identified as competitive business practices, poor ethical decision making, corruption in the government, tax regulation, confused policy, excessive bureaucracy, lack of executive commitment and unprofessional management, and inadequate evaluation of CSR initiatives. The most significant obstacles are those related to lack of resources - training-related, financial, and informational.

Few studies have so far attempted to analyze CSR issues in Indian textile sectors with respect to CSR perceptions amongst consumers [Gupta and Hodges (2012)] ; Organisational culture and environmental responsibility [Dash et al.(2009)] ; sustainable supplier assessment (Baskaran(2011)) . Some studies particularly analyze the barriers of CSR implementation with a national perspective. 
For instance, Valmohammadi(2011) explored the understandings of a CSR domain and concepts in an Iranian context and particularly focused on a code of conduct named "ISO 26000. He addressed the seven core issues while implementing CSR . In a similar study amongst Bnagladeshi managers, Duarte and Rahman studied the CSR with a view of managers's perspective . Arevalo and Aravind (2011) explored the practices of CSR in an Indian context and determined four types of CSR approaches: the ethical, the statist, the liberal, and the stakeholder. Monte and Leire (2013) investigated various CSR barriers with respect to supply chain in Swedish industries. Garavan et al. (2011) analyzed the behavioral barriers of CSR and corporate sustainability (CS) with the concern of human resource development. Chi (2010) analyzed the development, achievements, and challenges of CSR practices in the Chinese textile and apparel industry. He also extended the topic with a consideration of supply chains. Cooke and He [29] connected the terms human resource management and corporate social responsibility in the Chinese textile and apparel industry through the perception of the managers regarding these two terms. Pedersen et al. (2013) investigated the institutional pressures of CSR within the Nordic fashion industry with the assistance of responses from 400 fashion companies in Denmark, Norway, Sweden, Finland, and Iceland. From this study, they finalized that the pressures are not central to those nations but rather they differ among various stakeholder groups. Goworek (2011) explored the social and environmental issues involved in the clothing industry through a case study adapted in UK. Kozar and Connell (2013) have analysed the CSR in apparel and textile related industry with respect to attitudes, knowledge and purchasing behaviour of consumer whereas a similar study is done by Dickson and Eckman (2006) from the perspectives of apparel and textile scholars . Baskaran et al. [2011,2012] finds that Indian textile organizations are still able to implement CSR successfully despite the pressure from various impediments such as soil contamination and health issues. Another business practice found too often in textile industries is forced overtime work. Because it is common for nations with higher populations to have fewer job opportunities, workers often feel forced to accept overtime work. The result is an increase in workers' personal tension, which may easily lead to great health risk.

To identify relevant studies on CSR drivers/barriers, a set of primary keywords such as CSR, sustainability, drivers/barriers, outcomes, concept and perception etc. for a computer search of the Science, Scopus and ScienceDirect databases. Only papers published in peer-review journals from 2001 onwards were included in the search. This study primarily focuses on Govindan et al. (2015) paper in identifying the common barriers in Indian textile industry. Some of the common barriers recognised were competitive business practices, poor ethical decision making, corruption in the government, tax regulation, confused policy, excessive bureaucracy, lack of executive commitment and unprofessional management, and inadequate evaluation of CSR initiatives. The most significant obstacles are those related to lack of resources - training-related, financial, and informational. Following table 1 identify common barriers to CSR implementation with respect to Indian textile industry along with their description [Valmohammadi , 2011 and Duarte and Rahman , 2010] .
Table 1 : Common barriers to CSR implementation in Indian textile industry

\begin{tabular}{|c|c|c|}
\hline $\begin{array}{l}\text { S. } \\
\text { No. }\end{array}$ & Barrier & Description \\
\hline 1. & $\begin{array}{c}\text { Absence of } \\
\text { stakeholder } \\
\text { awareness } \\
\text { (ASA) }\end{array}$ & $\begin{array}{l}\text { In developing countries, it is quite } \\
\text { common that stakeholders are not } \\
\text { aware of the benefits of CSR. }\end{array}$ \\
\hline 2. & $\begin{array}{c}\text { Absence of } \\
\text { appropriate } \\
\text { training (AAT) }\end{array}$ & $\begin{array}{c}\text { CSR implementation requires an } \\
\text { appropriate training on part of an } \\
\text { organisations employees and managers }\end{array}$ \\
\hline 3. & $\begin{array}{l}\text { Absence of } \\
\text { knowledge and } \\
\text { information } \\
(\mathrm{AKI})\end{array}$ & $\begin{array}{l}\text { Because CSR is potentially difficult to } \\
\text { define, acquiring relevant information } \\
\text { regarding CSR presents challenges and } \\
\text { slows down effective implementation. }\end{array}$ \\
\hline 4. & $\begin{array}{l}\text { Budgetary } \\
\text { constraints } \\
\quad \text { (BC) }\end{array}$ & $\begin{array}{l}\text { CSR implementation requires high } \\
\text { initial investment . Therefore, } \\
\text { budgetary constraint becomes an barrier } \\
\text { in developing countries. }\end{array}$ \\
\hline 5. & $\begin{array}{l}\text { Absence of } \\
\text { required level } \\
\text { of consumer } \\
\text { willingness and } \\
\text { awareness } \\
\text { (ACA) }\end{array}$ & $\begin{array}{l}\text { Indian customers are more likely to go } \\
\text { for economical profit rather than } \\
\text { quality and other societal parameters. } \\
\text { This motivates the manufacturers to } \\
\text { focus on profits only. }\end{array}$ \\
\hline 6. & $\begin{array}{l}\text { Absence of } \\
\text { motivation to } \\
\text { build } \\
\text { reputation } \\
\text { (AMR) }\end{array}$ & $\begin{array}{l}\text { Usually small scale and developing } \\
\text { organisations do not realize the value of } \\
\text { their reputation. As a result, they are } \\
\text { not motivated to implement CSR. }\end{array}$ \\
\hline 7. & $\begin{array}{l}\text { Lack of } \\
\text { regulations and } \\
\text { standards } \\
(\text { LRS })\end{array}$ & $\begin{array}{c}\text { Laws are not clearly or weakly } \\
\text { established in developing nations as } \\
\text { compared to European or US countries } \\
\text { which freely allows the stakeholders to } \\
\text { avoid the CSR. }\end{array}$ \\
\hline 8. & $\begin{array}{c}\text { Diversity or } \\
\text { socio cultural } \\
\text { barriers (SCB) }\end{array}$ & $\begin{array}{l}\text { Diversity or socio-cultural barriers } \\
\text { restricts the practitioners from } \\
\text { understanding how to implement the } \\
\text { CSR based on their origin. }\end{array}$ \\
\hline 9. & $\begin{array}{c}\text { Company's } \\
\text { values and } \\
\text { culture (CVC) }\end{array}$ & $\begin{array}{l}\text { Some organizations, relying on old } \\
\text { company culture, are resistant to } \\
\text { changing to new strategies like CSR }\end{array}$ \\
\hline 10. & $\begin{array}{c}\text { Lack of social } \\
\text { audit (LSA) }\end{array}$ & $\begin{array}{l}\text { Lack of social audit is one of the } \\
\text { barriers for CSR which allows the } \\
\text { stakeholders to be exempt from CSR } \\
\text { reporting }\end{array}$ \\
\hline 11. & $\begin{array}{l}\text { Lack of top } \\
\text { management } \\
\text { commitment } \\
(\text { LMC) }\end{array}$ & $\begin{array}{l}\text { Some of the top level managers only } \\
\text { focus on financial profit rather than } \\
\text { societal benefit }\end{array}$ \\
\hline 12. & $\begin{array}{l}\text { Lack of market } \\
\text { or professional } \\
\text { visibility } \\
\text { (LMV) }\end{array}$ & $\begin{array}{c}\text { Usually CSR implementation allows an } \\
\text { organisation to showcase itself in } \\
\text { national as well as international } \\
\text { markets through publication of } \\
\text { sustainability reports [Kronenberg, } \\
\text { 2012], alignment with foreign partners } \\
\text { [Lewicka, 2006], international } \\
\text { diversification [Cheung et al. , 2015] } \\
\text { etc. }\end{array}$ \\
\hline
\end{tabular}




\begin{tabular}{|c|c|c|}
\hline 13. & $\begin{array}{c}\text { Poor ethical } \\
\text { decision } \\
\text { making } \\
\text { (PEDM) }\end{array}$ & $\begin{array}{c}\text { Lack of management commitment and } \\
\text { interest often results in poor ethical } \\
\text { decision making }\end{array}$ \\
\hline 14. & $\begin{array}{c}\text { Environmental } \\
\text { concerns (EC) }\end{array}$ & $\begin{array}{c}\text { This includes soil contamination and } \\
\text { health defects }\end{array}$ \\
\hline 15. & $\begin{array}{c}\text { Forced } \\
\text { overtime work } \\
\text { ( FOW) }\end{array}$ & $\begin{array}{c}\text { Usually CSR implementation requires } \\
\text { extra or overtime to meet the required } \\
\text { targets. This may be unacceptable by } \\
\text { workers in case of tight budgetary } \\
\text { constraints }\end{array}$ \\
\hline 16. & $\begin{array}{c}\text { Lack of } \\
\text { government } \\
\text { willingness } \\
\text { (LGW) and } \\
\text { support }\end{array}$ & $\begin{array}{c}\text { Lack or disinterest on part of a } \\
\text { country's government often is one of } \\
\text { the major barrier as government plays a } \\
\text { very important role in any nations' } \\
\text { success }\end{array}$ \\
\hline \multicolumn{2}{|c}{} \\
\hline
\end{tabular}

\section{INTERPRETIVE STRUCTURAL MODELLING METHODOLOGY}

1. Identification of elements, which are relevant to the decision maker's problems and issues.

2. Establishing the contextual relationship between elements and with respect to which pairs of elements will be examine.

3. A structural self-interaction matrix (SSIM) may be developed between two variables i.e. $i$ and $j$. It establishes the "Lead to" relationship between criteria. Four symbols viz. V, A, X \& O are used for establishing the relationships.

4. SSIM may be further used to develop an initial reachability matrix which has all values in binary form. Rule of transitivity is usually checked at this stage. After removing the transitivity, final reachability matrix will form.

5. Afterwards, the reachability set and antecedent set for each criterion and for each element can be obtained from the final reachability matrix.

6. After that a level partition matrix can be obtained based on establishing the precedence relationships and arranging the elements in a topological order.

7. A Mic-Mac analysis can be performed which categorize the variables as per the driving and dependence power in to autonomous, dependent, driver and linkage category.

8. Finally a diagraph can be obtained.

\section{DEVELOPMENT OF ISM MODEL}

In this section, ISM model is developed for studying the interrelationships amongst various barriers to to CSR implementation in Indian textile industry. The sixteen barriers recognized are : Absence of stakeholder awareness (ASA) ; Absence of appropriate training (AAT) ; Absence of knowledge and information (AKI) ; Budgetary constraints (BC); Absence of required level of consumer awareness (ACA); Absence of motivation to build reputation (AMR) ; Lack of regulations and standards (LRS); Diversity or sociocultural barriers (SCB) ; Company's values and culture (CVC) ; Lack of social audit (LSA); Lack of top management commitment (LMC); Lack of market or professional visibility (LMV); Poor ethical decision making (PEDM);
Environmental concerns (EC); Forced overtime work (FOW) ; Lack of government willingness (LGW).

\subsection{Construction of Structural Self - Interaction Matrix (SSIM)}

This matrix gives the pair-wise relationship between two variables i.e. $\mathrm{i}$ and $\mathrm{j}$ based on VAXO. SSIM has been presented below in Fig 1.

\subsection{Construction of Initial Reachability Matrix and final reachability matrix}

The SSIM has been converted in to a binary matrix called the initial reachability matrix shown in fig. 2 by substituting V, A, $\mathrm{X}, \mathrm{O}$ by 1 or 0 as per the case. After incorporating the transitivity, the final reachability matrix is shown below in the Fig 3.

\subsection{Level Partition}

From the final reachability matrix, reachability and final antecedent set for each factor are found. The elements for which the reachability and intersection sets are same are the top-level element in the ISM hierarchy. After the identification of top level element, it is separated out from the other elements and the process continues for next level of elements. Reachability set, antecedent set, intersection set along with different level for elements have been shown below in table 2 to table 12 .

Table 2 Iteration I

\begin{tabular}{|c|c|c|c|c|}
\hline S.No. & $\begin{array}{c}\text { Reachability } \\
\text { set }\end{array}$ & $\begin{array}{l}\text { Antecedent } \\
\text { set }\end{array}$ & $\begin{array}{c}\text { Intersectio } \\
\text { n set }\end{array}$ & $\begin{array}{l}\text { Itera } \\
\text { tion }\end{array}$ \\
\hline 1 & 14 & $\begin{array}{c}1,2,3,4,5,6,7,8, \\
9,10,11,12,13, \\
14,15,16\end{array}$ & 14 & \multirow{11}{*}{$\mathbf{I}$} \\
\hline 2 & 14,15 & $\begin{array}{c}1,2,3,4,5,67,8 \\
9,10,11,12,13 \\
15,16\end{array}$ & 15 & \\
\hline 3 & $\begin{array}{c}6,7,10,13,14 \\
15\end{array}$ & $\begin{array}{c}1,2,3,4,5,6,7,8, \\
9,10,11,12,13, \\
16\end{array}$ & $6,7,10,13$ & \\
\hline 4 & $\begin{array}{c}6,7,10,12,13 \\
14,15\end{array}$ & $\begin{array}{c}1,2,3,4,5,6,7,8 \\
9,11,12,13,16\end{array}$ & $6,7,12,13$ & \\
\hline 5 & $\begin{array}{c}2,5,6,7,10,12 \\
13,14,15\end{array}$ & $\begin{array}{c}1,2,3,4,5,6,7,8 \\
9,11,12,16\end{array}$ & $2,5,6,7,12$ & \\
\hline 6 & $\begin{array}{c}2,3,5,6,7,10,12, \\
13,14,15\end{array}$ & $\begin{array}{c}1,2,3,4,5,7,8,9 \\
11,12,16\end{array}$ & $2,3,5,7,12$ & \\
\hline 7 & $\begin{array}{c}2,3,5,6,7,10,11 \\
12,13,14,15\end{array}$ & $\begin{array}{c}1,2,3,4,5,7,9,1 \\
1,12,16\end{array}$ & $\begin{array}{c}2,3,5,7,11, \\
12\end{array}$ & \\
\hline 8 & $\begin{array}{c}1,2,3,5,6,7,10,1 \\
1,12,13,14,15\end{array}$ & $1,4,5,9,11,16$ & $1,5,11$ & \\
\hline 9 & $\begin{array}{c}1,2,3,5,6,7,8 \\
10,11,12,13,14 \\
15\end{array}$ & $1,4,9,11,16$ & 1,11 & \\
\hline 10 & $\begin{array}{c}1,2,3,4,5,6,7,8 \\
10,11,12,13,14 \\
15\end{array}$ & $4,9,16$ & 4 & \\
\hline 11 & $\begin{array}{c}1,2,3,4,5,6,7,8 \\
10,11,12,13,14 \\
15,16\end{array}$ & 9,16 & 16 & \\
\hline
\end{tabular}


Fig 1: Structural Self- Interaction Matrix

\begin{tabular}{|c|c|c|c|c|c|c|c|c|c|c|c|c|c|c|c|c|c|}
\hline Barrie & & 1 & 2 & 3 & 4 & 5 & 6 & 7 & 8 & 9 & 10 & 11 & 12 & 13 & 14 & 15 & 16 \\
\hline & & $\begin{array}{c}\text { AS } \\
\text { A }\end{array}$ & $\begin{array}{c}\text { AA } \\
\text { T }\end{array}$ & $\begin{array}{c}\mathrm{AK} \\
\mathrm{I}\end{array}$ & $\mathrm{BC}$ & ACA & $\begin{array}{c}\mathrm{AM} \\
\mathrm{R}\end{array}$ & $\begin{array}{c}\text { LR } \\
\text { S }\end{array}$ & $\begin{array}{l}\mathrm{S} \\
\mathrm{C} \\
\mathrm{B}\end{array}$ & $\begin{array}{c}\text { CV } \\
\mathrm{C}\end{array}$ & LSA & $\begin{array}{c}\mathrm{LM} \\
\mathrm{C}\end{array}$ & $\begin{array}{c}\text { LM } \\
\text { V }\end{array}$ & $\begin{array}{l}\text { PE } \\
\text { DM }\end{array}$ & EC & $\begin{array}{c}\text { FO } \\
\text { W }\end{array}$ & $\begin{array}{c}\text { LG } \\
\text { W }\end{array}$ \\
\hline 1. & ASA & & V & V & A & X & V & V & A & A & $\mathrm{X}$ & A & V & X & V & $\mathrm{O}$ & A \\
\hline 2. & AAT & & & X & A & $\mathrm{O}$ & A & A & A & A & V & A & $X$ & V & V & A & A \\
\hline 3. & AKI & & & & A & V & V & V & A & A & V & A & $\mathrm{X}$ & V & V & V & A \\
\hline 4. & $\mathrm{BC}$ & & & & & V & V & V & V & A & V & $\mathrm{V}$ & V & V & V & V & A \\
\hline 5. & $\mathrm{ACA}$ & & & & & & $X$ & V & A & A & V & A & V & V & V & V & A \\
\hline 6. & AMR & & & & & & & A & A & A & V & A & V & V & V & $\mathrm{O}$ & A \\
\hline 7. & LRS & & & & & & & & A & A & V & A & V & A & V & V & A \\
\hline 8. & SCB & & & & & & & & & A & V & A & V & V & V & V & A \\
\hline 9. & $\mathrm{CVC}$ & & & & & & & & & & $\mathrm{V}$ & $\mathrm{V}$ & V & V & V & V & V \\
\hline 10. & LSA & & & & & & & & & & & A & A & V & V & V & A \\
\hline 11. & LMC & & & & & & & & & & & & $X$ & V & V & V & $\mathrm{A}$ \\
\hline 12. & LMV & & & & & & & & & & & & & V & V & $\mathrm{O}$ & A \\
\hline 13. & PEDM & & & & & & & & & & & & & & $\mathrm{V}$ & $\mathrm{V}$ & A \\
\hline 14. & EC & & & & & & & & & & & & & & & A & A \\
\hline 15. & FOW & & & & & & & & & & & & & & & & A \\
\hline 16. & LGW & & & & & & & & & & & & & & & & \\
\hline
\end{tabular}

Fig 2: Initial reachability matrix

\begin{tabular}{|c|c|c|c|c|c|c|c|c|c|c|c|c|c|c|c|c|c|}
\hline Barrie & & 1 & 2 & 3 & 4 & 5 & 6 & 7 & 8 & 9 & 10 & 11 & 12 & 13 & 14 & 15 & 16 \\
\hline & & $\begin{array}{c}\text { AS } \\
\text { A }\end{array}$ & $\begin{array}{c}\text { AA } \\
\text { T }\end{array}$ & $\begin{array}{c}\text { AK } \\
\text { I }\end{array}$ & $\mathrm{BC}$ & ACA & $\begin{array}{c}\mathrm{AM} \\
\mathrm{R}\end{array}$ & $\begin{array}{c}\text { LR } \\
\text { S }\end{array}$ & $\begin{array}{l}\mathrm{S} \\
\mathrm{C} \\
\mathrm{B}\end{array}$ & $\begin{array}{c}\mathrm{CV} \\
\mathrm{C}\end{array}$ & LSA & $\begin{array}{c}\text { LM } \\
\text { C }\end{array}$ & $\begin{array}{c}\text { LM } \\
\text { V }\end{array}$ & $\begin{array}{l}\text { PE } \\
\mathrm{DM}\end{array}$ & $\mathrm{EC}$ & $\begin{array}{l}\text { FO } \\
W\end{array}$ & $\begin{array}{c}\text { LG } \\
W\end{array}$ \\
\hline 1. & ASA & 1 & 1 & 1 & 0 & 1 & 1 & 1 & 0 & 0 & 1 & 0 & 1 & 1 & 1 & 0 & 0 \\
\hline 2. & AAT & 0 & 1 & 1 & 0 & 0 & 0 & 0 & 0 & 0 & 1 & 0 & 1 & 1 & 1 & 0 & 0 \\
\hline 3. & AKI & 0 & 1 & 1 & 0 & 1 & 1 & 1 & 0 & 0 & 1 & 0 & 1 & 1 & 1 & 1 & 0 \\
\hline 4. & $\mathrm{BC}$ & 1 & 1 & 1 & 1 & 1 & 1 & 1 & 1 & 0 & 1 & 1 & 1 & 1 & 1 & 1 & 0 \\
\hline 5. & ACA & 1 & 0 & 0 & 0 & 1 & 1 & 1 & 0 & 0 & 1 & 0 & 1 & 1 & 1 & 1 & 0 \\
\hline 6. & AMR & 0 & 1 & 0 & 0 & 1 & 1 & 0 & 0 & 0 & 1 & 0 & 1 & 1 & 1 & 0 & 0 \\
\hline 7. & LRS & 0 & 1 & 0 & 0 & 0 & 1 & 1 & 0 & 0 & 1 & 0 & 1 & 0 & 1 & 1 & 0 \\
\hline 8. & SCB & 1 & 1 & 1 & 0 & 1 & 1 & 1 & 1 & 0 & 1 & 0 & 1 & 1 & 1 & 1 & 0 \\
\hline 9. & $\mathrm{CVC}$ & 1 & 1 & 1 & 1 & 1 & 1 & 1 & 1 & 1 & 1 & 1 & 1 & 1 & 1 & 1 & 1 \\
\hline 10. & LSA & 1 & 0 & 0 & 0 & 0 & 0 & 0 & 0 & 0 & 1 & 0 & 0 & 1 & 1 & 1 & 0 \\
\hline 11. & LMC & 1 & 1 & 1 & 0 & 1 & 1 & 1 & 1 & 0 & 1 & 1 & 1 & 1 & 1 & 1 & 0 \\
\hline 12. & LMV & 0 & 1 & 1 & 0 & 0 & 0 & 0 & 0 & 0 & 1 & 1 & 1 & 1 & 1 & 0 & 0 \\
\hline 13. & PEDM & 1 & 0 & 0 & 0 & 0 & 0 & 1 & 0 & 0 & 0 & 0 & 0 & 1 & 1 & 1 & 0 \\
\hline 14. & $\mathrm{EC}$ & 0 & 0 & 0 & 0 & 0 & 0 & 0 & 0 & 0 & 0 & 0 & 0 & 0 & 1 & 0 & 0 \\
\hline 15. & FOW & 0 & 1 & 0 & 0 & 0 & 0 & 0 & 0 & 0 & 0 & 0 & 0 & 0 & 1 & 1 & 0 \\
\hline 16. & LGW & 1 & 1 & 1 & 1 & 1 & 1 & 1 & 1 & 0 & 1 & 1 & 1 & 1 & 1 & 1 & 1 \\
\hline
\end{tabular}


Fig 3 : Final reachability matrix

\begin{tabular}{|c|c|c|c|c|c|c|c|c|c|c|c|c|c|c|c|c|c|c|}
\hline Barrie & & 1 & 2 & 3 & 4 & 5 & 6 & 7 & 8 & 9 & 10 & 11 & 12 & 13 & 14 & 15 & 16 & \\
\hline & & $\begin{array}{c}\text { AS } \\
\text { A }\end{array}$ & $\begin{array}{c}\text { AA } \\
\text { T }\end{array}$ & $\begin{array}{c}\text { AK } \\
\text { I }\end{array}$ & $\mathrm{BC}$ & $\begin{array}{c}\mathrm{AC} \\
\mathrm{A}\end{array}$ & $\begin{array}{c}\mathrm{AM} \\
\mathrm{R}\end{array}$ & LRS & $\begin{array}{c}\text { SC } \\
\text { B }\end{array}$ & $\begin{array}{l}\mathrm{C} \\
\mathrm{V} \\
\mathrm{C}\end{array}$ & $\begin{array}{c}\text { LS } \\
\text { A }\end{array}$ & $\begin{array}{c}\mathrm{LM} \\
\mathrm{C}\end{array}$ & $\begin{array}{c}\text { LM } \\
\text { V }\end{array}$ & $\begin{array}{c}\mathrm{PE} \\
\mathrm{D} \\
\mathrm{M}\end{array}$ & $\mathrm{EC}$ & $\begin{array}{l}\text { FO } \\
\text { W }\end{array}$ & $\begin{array}{c}\text { LG } \\
\text { W }\end{array}$ & D.P \\
\hline 1. & ASA & 1 & 1 & 1 & 0 & 1 & 1 & 1 & 0 & 0 & 1 & 1 & 1 & 1 & 1 & 1 & 0 & 12 \\
\hline 2. & AAT & 0 & 1 & 1 & 0 & 1 & 1 & 1 & 0 & 0 & 1 & 1 & 1 & 1 & 1 & 1 & 0 & 11 \\
\hline 3. & AKI & 0 & 1 & 1 & 0 & 1 & 1 & 1 & 0 & 0 & 1 & 1 & 1 & 1 & 1 & 1 & 0 & 11 \\
\hline 4. & BC & 1 & 1 & 1 & 1 & 1 & 1 & 1 & 1 & 0 & 1 & 1 & 1 & 1 & 1 & 1 & 0 & 14 \\
\hline 5. & ACA & 1 & 1 & 1 & 0 & 1 & 1 & 1 & 0 & 0 & 1 & 1 & 1 & 1 & 1 & 1 & 0 & 9 \\
\hline 6. & AMR & 0 & 1 & 0 & 0 & 1 & 1 & 1 & 0 & 0 & 1 & 0 & 1 & 1 & 1 & 1 & 0 & 9 \\
\hline 7. & LRS & 0 & 1 & 1 & 0 & 1 & 1 & 1 & 0 & 0 & 1 & 1 & 1 & 1 & 1 & 1 & 0 & 11 \\
\hline 8. & SCB & 1 & 1 & 1 & 0 & 1 & 1 & 1 & 1 & 0 & 1 & 1 & 1 & 1 & 1 & 1 & 0 & 12 \\
\hline 9. & CVC & 1 & 1 & 1 & 1 & 1 & 1 & 1 & 1 & 1 & 1 & 1 & 1 & 1 & 1 & 1 & 1 & 16 \\
\hline 10. & LSA & 1 & 0 & 0 & 0 & 0 & 1 & 1 & 0 & 0 & 1 & 0 & 0 & 1 & 1 & 1 & 0 & 7 \\
\hline 11. & LMC & 1 & 1 & 1 & 0 & 1 & 1 & 1 & 1 & 0 & 1 & 1 & 1 & 1 & 1 & 1 & 0 & 13 \\
\hline 12. & LMV & 0 & 1 & 1 & 0 & 1 & 1 & 1 & 1 & 0 & 1 & 1 & 1 & 1 & 1 & 1 & 0 & 11 \\
\hline 13. & PEDM & 1 & 0 & 0 & 0 & 0 & 1 & 1 & 0 & 0 & 1 & 0 & 1 & 1 & 1 & 1 & 0 & 9 \\
\hline 14. & EC & 0 & 0 & 0 & 0 & 0 & 0 & 0 & 0 & 0 & 0 & 0 & 0 & 0 & 1 & 0 & 0 & 1 \\
\hline 15. & FOW & 0 & 1 & 0 & 0 & 0 & 0 & 0 & 0 & 0 & 0 & 0 & 0 & 0 & 1 & 1 & 0 & 3 \\
\hline \multirow[t]{2}{*}{16.} & LGW & 1 & 1 & 1 & 1 & 1 & 1 & 1 & 1 & 0 & 1 & 1 & 1 & 1 & 1 & 1 & 1 & 15 \\
\hline & De.P & 9 & 13 & 11 & 3 & 12 & 14 & 14 & 6 & 1 & 14 & 11 & 13 & 14 & 16 & 15 & 2 & \\
\hline
\end{tabular}

D.P : Driving power ; De.P : dependence power

Table 3 : Iteration II

\begin{tabular}{|c|c|c|c|c|}
\hline $\begin{array}{l}\text { Sr. } \\
\text { No. }\end{array}$ & $\begin{array}{c}\text { Reachability } \\
\text { set }\end{array}$ & Antecedent set & $\begin{array}{c}\text { Intersect } \\
\text { ion set }\end{array}$ & $\begin{array}{l}\text { Itera } \\
\text { tion }\end{array}$ \\
\hline 2 & 15 & $\begin{array}{c}1,2,3,4,5,67,8,9, \\
10,11,12,13,15,16\end{array}$ & 15 & \\
\hline 3 & $6,7,10,13,15$ & $\begin{array}{c}1,2,3,4,5,6,7,8,9 \\
10,11,12,13,16\end{array}$ & $\begin{array}{c}6,7,10 \\
13\end{array}$ & \\
\hline 4 & $\begin{array}{c}6,7,10,12,13 \\
15\end{array}$ & $\begin{array}{c}1,2,3,4,5,6,7,8,9 \\
11,12,13,16\end{array}$ & $\begin{array}{c}6,7,12 \\
13\end{array}$ & \\
\hline 5 & $\begin{array}{c}2,5,6,7,10 \\
12,13,15\end{array}$ & $\begin{array}{c}1,2,3,4,5,6,7,8,9 \\
11,12,16\end{array}$ & $\begin{array}{c}2,5,6,7 \\
12\end{array}$ & \\
\hline 6 & $\begin{array}{c}2,3,5,6,7,10 \\
12,13,15\end{array}$ & $\begin{array}{c}1,2,3,4,5,7,8,9,11,1 \\
2,16\end{array}$ & $\begin{array}{c}2,3,5,7 \\
12\end{array}$ & \\
\hline 7 & $\begin{array}{l}2,3,5,6,7,10 \\
11,12,13,15\end{array}$ & $\begin{array}{c}1,2,3,4,5,7,9,11, \\
12,16\end{array}$ & $\begin{array}{c}2,3,5,7 \\
11,12\end{array}$ & \\
\hline 8 & $\begin{array}{c}1,2,3,5,6,7 \\
10,11,12,13 \\
15\end{array}$ & $1,4,5,9,11,16$ & $1,5,11$ & II \\
\hline 9 & $1,2,3,5,6,7,8$ & $1,4,9,11,16$ & 1,11 & \\
\hline
\end{tabular}

\begin{tabular}{|c|c|c|c|}
\hline & $\begin{array}{c}10,11,12,13 \\
15\end{array}$ & & \\
\hline 10 & $\begin{array}{c}1,2,3,4,5,6,7,8 \\
10,11,12,13 \\
15\end{array}$ & $4,9,16$ & 4 \\
\hline 11 & $\begin{array}{c}1,2,3,4,5,6,7,8 \\
10,11,12,13, \\
15,16\end{array}$ & 9,16 & 16 \\
\hline
\end{tabular}

Table 4 : Iteration III

\begin{tabular}{|c|c|c|c|c|}
\hline $\begin{array}{l}\text { Sr. } \\
\text { No. }\end{array}$ & Reachability set & Antecedent set & $\begin{array}{c}\text { Intersectio } \\
\text { n set }\end{array}$ & $\begin{array}{l}\text { Itera } \\
\text { tion }\end{array}$ \\
\hline 3 & $6,7,10,13$ & $\begin{array}{c}1,2,3,4,5,6,7,8, \\
9,10,11,12,13,1 \\
6\end{array}$ & $6,7,10,13$ & \\
\hline 4 & $6,7,10,12,13$ & $\begin{array}{c}1,2,3,4,5,6,7,8 \\
9,11,12,13,16\end{array}$ & $6,7,12,13$ & \\
\hline 5 & $2,5,6,7,10,12,13$ & $\begin{array}{c}1,2,3,4,5,6,7,8 \\
9,11,12,16\end{array}$ & $2,5,6,7,12$ & \\
\hline 6 & $\begin{array}{c}2,3,5,6,7,10,12,1 \\
3\end{array}$ & $\begin{array}{c}1,2,3,4,5,7,8,9 \\
11,12,16\end{array}$ & $2,3,5,7,12$ & \\
\hline
\end{tabular}




\begin{tabular}{|c|c|c|c|}
\hline 7 & $\begin{array}{c}2,3,5,6,7,10,11,1 \\
2,13\end{array}$ & $\begin{array}{c}1,2,3,4,5,7,9,11 \\
, 12,16\end{array}$ & $\begin{array}{c}2,3,5,7,11, \\
12\end{array}$ \\
\hline 8 & $\begin{array}{c}1,2,3,5,6,7,10,11 \\
12,13\end{array}$ & $1,4,5,9,11,16$ & $1,5,11$ \\
\hline 9 & $\begin{array}{c}1,2,3,5,6,7,8,10,1 \\
1, \\
12,13\end{array}$ & $1,4,9,11,16$ & 1,11 \\
\hline 10 & $\begin{array}{c}1,2,3,4,5,6,7,8 \\
10,11,12,13\end{array}$ & $4,9,16$ & 4 \\
\hline 11 & $\begin{array}{l}1,2,3,4,5,6,7,8, \\
10,11,12,13,16\end{array}$ & 9,16 & 16 \\
\hline 12 & $\begin{array}{c}1,2,3,4,5,6,7,8,9 \\
10,11,12,13,16\end{array}$ & 9 & 9 \\
\hline
\end{tabular}

Table 5 : Iteration IV

\begin{tabular}{|c|c|c|c|c|}
\hline $\begin{array}{l}\text { S. } \\
\text { No }\end{array}$ & Reachability set & $\begin{array}{c}\text { Antecedent } \\
\text { set }\end{array}$ & $\begin{array}{c}\text { Intersectio } \\
\text { n set }\end{array}$ & $\begin{array}{c}\text { Iteratio } \\
\mathbf{n} / \\
\text { Levels }\end{array}$ \\
\hline 4 & 12 & $\begin{array}{c}1,2,3,4,5,8,9 \\
11,12,16\end{array}$ & 12 & \multirow{9}{*}{ IV } \\
\hline 5 & $2,5,12$ & $\begin{array}{c}1,2,3,4,5,8,9 \\
11,12,16\end{array}$ & $2,5,12$ & \\
\hline 6 & $2,3,5,12$ & $\begin{array}{c}1,2,3,4,5,8,9 \\
11,12,16\end{array}$ & $2,3,5,12$ & \\
\hline 7 & $2,3,5,11,12$ & $\begin{array}{c}1,2,3,4,5,9 \\
11,12,16\end{array}$ & $2,3,5,11,12$ & \\
\hline 8 & $1,2,3,5,11,12$ & $1,4,5,9,11,16$ & $1,5,11$ & \\
\hline 9 & $1,2,3,5,8,11,12$ & $1,4,9,11,16$ & 1,11 & \\
\hline 10 & $\begin{array}{c}1,2,3,4,5,8, \\
11,12\end{array}$ & $4,9,16$ & 4 & \\
\hline 11 & $\begin{array}{c}1,2,3,4,5,8 \\
11,12,16\end{array}$ & 9,16 & 16 & \\
\hline 12 & $\begin{array}{c}1,2,3,4,5,8,9,11,1 \\
2,16\end{array}$ & 9 & 9 & \\
\hline
\end{tabular}

Table 6 : Iteration V

\begin{tabular}{|c|c|c|c|c|}
\hline $\begin{array}{c}\text { Sr. } \\
\text { No } \\
\cdot\end{array}$ & $\begin{array}{c}\text { Reachability } \\
\text { set }\end{array}$ & Antecedent set & $\begin{array}{c}\text { Intersection } \\
\text { set }\end{array}$ & $\begin{array}{c}\text { Itera } \\
\text { tion }\end{array}$ \\
\hline 6 & 3 & $1,2,3,4,8,9,11,16$ & 3 & \\
\hline 7 & $\mathbf{3 , 1 1}$ & $1,2,3,4,8,9,11,16$ & 3,11 & \multirow{2}{*}{$\mathbf{V}$} \\
\hline 8 & $1,3,11$ & $1,4,9,8,11,16$ & 1,11 & \\
\hline 9 & $1,3,8,11$ & $1,4,8,9,11,16$ & 1,11 & \\
\hline 10 & $1,3,4,8,11$ & $4,9,16$ & 4 & \\
\hline 11 & $1,3,4,8,11,16$ & 9,16 & 16 & \\
\hline 12 & $1,3,4,8,9,11$, & 9 & 9 & \\
& 16 & & &
\end{tabular}

Table 7 : Iteration VI

\begin{tabular}{|c|c|c|c|c|}
\hline $\begin{array}{c}\text { Sr. } \\
\text { No. }\end{array}$ & $\begin{array}{c}\text { Reachability } \\
\text { set }\end{array}$ & $\begin{array}{c}\text { Antecedent } \\
\text { set }\end{array}$ & $\begin{array}{c}\text { Intersection } \\
\text { set }\end{array}$ & $\begin{array}{c}\text { Itera } \\
\text { tion }\end{array}$ \\
\hline 8 & 1 & $1,4,8,9,16$ & 1 & \\
\cline { 1 - 4 } & $\mathbf{1 , 8}$ & $1,4,8,9,16$ & 1,8 & \multirow{2}{*}{ VI } \\
\cline { 1 - 3 } 10 & $1,4,8$ & $4,9,16$ & 4 & \\
\hline 11 & $1,4,8,16$ & 9,16 & 16 & \\
\hline 12 & $1,4,8,9,16$ & 9 & 9 & \\
\hline
\end{tabular}

Table 8 : Iteration VII

\begin{tabular}{|c|c|c|c|c|}
\hline $\begin{array}{c}\text { Sr. } \\
\text { No. }\end{array}$ & $\begin{array}{c}\text { Reachability } \\
\text { set }\end{array}$ & $\begin{array}{c}\text { Antecedent } \\
\text { set }\end{array}$ & $\begin{array}{c}\text { Intersection } \\
\text { set }\end{array}$ & $\begin{array}{c}\text { Itera } \\
\text { tion }\end{array}$ \\
\hline 10 & $\mathbf{4}$ & $4,9,16$ & 4 & \\
& VII \\
\hline 11 & 4,16 & 9,16 & 16 & \\
\hline 12 & $4,9,16$ & 9 & 9 & \\
\hline
\end{tabular}

Table 11 : Iteration VIII

\begin{tabular}{|c|c|c|c|c|}
\hline $\begin{array}{c}\text { Sr. } \\
\text { No. }\end{array}$ & $\begin{array}{c}\text { Reachability } \\
\text { set }\end{array}$ & $\begin{array}{c}\text { Antecedent } \\
\text { set }\end{array}$ & $\begin{array}{c}\text { Intersection } \\
\text { set }\end{array}$ & $\begin{array}{c}\text { Itera } \\
\text { tion }\end{array}$ \\
\hline 11 & $\mathbf{1 6}$ & 9,16 & 16 & VIII \\
\hline 12 & 9,16 & 9 & 9 & \\
\hline
\end{tabular}

Table 12 : Iteration IX

\begin{tabular}{|c|c|c|c|c|}
\hline $\begin{array}{c}\text { Sr. } \\
\text { No. }\end{array}$ & $\begin{array}{c}\text { Reachability } \\
\text { set }\end{array}$ & $\begin{array}{c}\text { Antecedent } \\
\text { set }\end{array}$ & $\begin{array}{c}\text { Intersection } \\
\text { set }\end{array}$ & $\begin{array}{c}\text { Itera } \\
\text { tion }\end{array}$ \\
\hline 12 & $\mathbf{9}$ & 9 & 9 & IX \\
\hline
\end{tabular}

\subsection{Classification of factors}

The critical success factors described earlier are classified in to four clusters viz. autonomous factor, dependent factors, linkage factors and independent factors. Fig. 4 below shows the driving power and dominance diagram

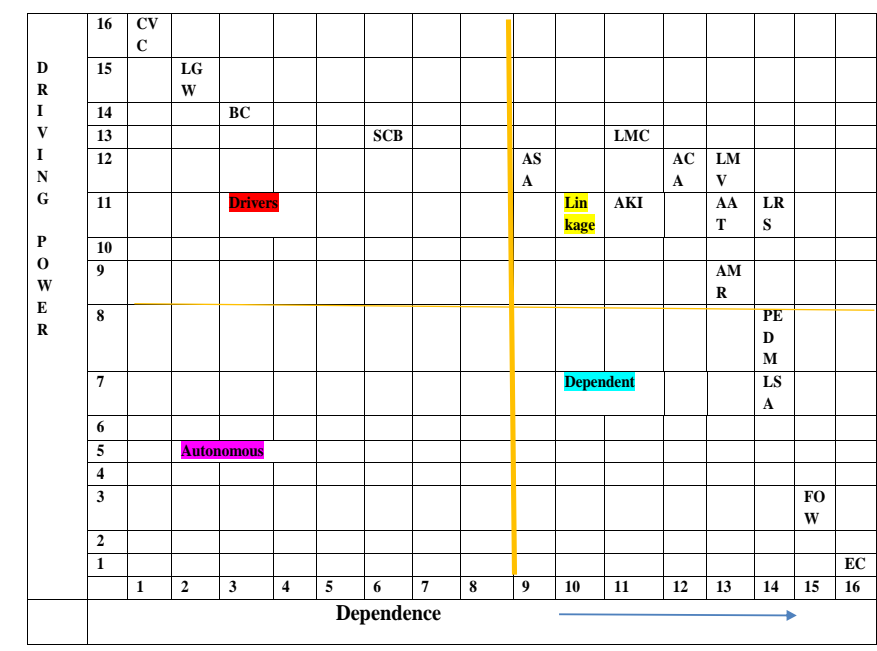

Fig . 4: Driving power and dependence diagram 


\subsection{ISM model}

An ISM model is developed ( as shown in fig. 5 below ) after arranging the elements as per their interaction or dependence relationships.

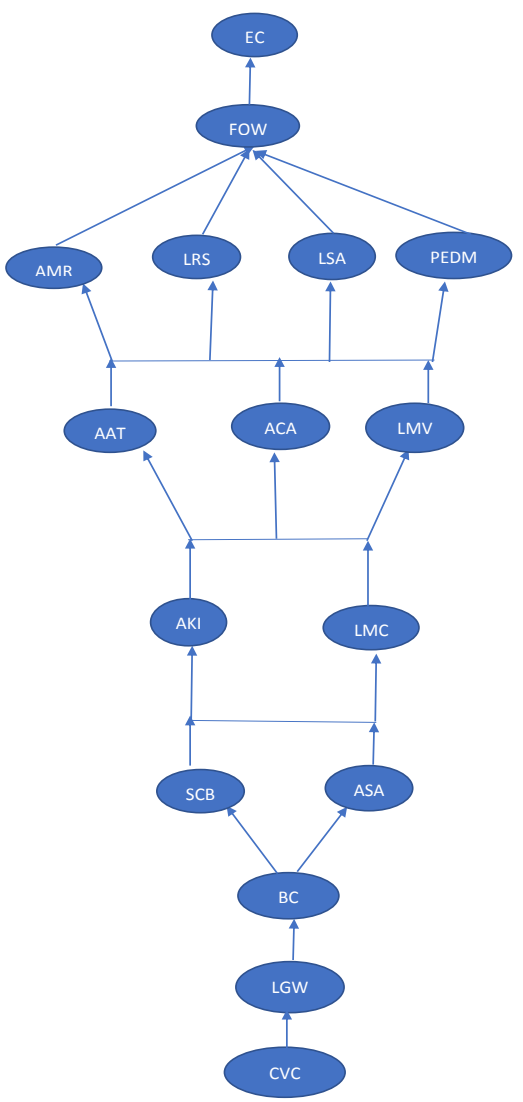

Fig 5: ISM diagraph

\section{CONCLUSIONS \& MANAGERIAL IMPLICATIONS}

1. Current research on CSR (Arevalo \& Aravind (2011) in India is mostly limited to either questionnaire surveys , consumer perceptions on CSR, attitude and behavioral studies, s corporate social reporting etc. studying the interrelationship amongst barriers and success factors towards CSR implementation could add a new paradigm in research .

2. A study of CSR outcomes with a focus on the Indian economy "could inform general managers as well as CSR managers about the attributes of the Indian approach to sustainability and CSR. This could be quite beneficial especially for a country that has the lowest level of GNP per capita and the highest level of CSR among other Asian economies (Reserve Bank of India, 2009; UNIDO, 2002).”

3. It is also hoped that organizations looking to work in India and indigenous to India will find the study beneficial in terms of factors which will lead to successfully meeting societal and environmental expectations, as well as lend a hand towards a more inclusive and equitable growth and development agenda.

\section{ACKNOWLEDGEMENTS}

Authors are thankful to Prof. S.P Singh, DMS , IIT Delhi for disseminating the knowledge on ISM methodology which has helped authors in writing this research work.

\section{REFERENCES}

[1] Shen , L. Kannan , G. and Shankar , M. 2015. Evaluation of Barriers of Corporate Social Responsibility Using an Analytical Hierarchy Process under a Fuzzy Environment-A Textile Case, Sustainability, 7, 34933514; doi:10.3390/su7033493

[2] Gupta, M.; Hodges, N. 2012. Corporate social responsibility in the apparel industry: An exploration of Indian consumers' perceptions and expectations. Journal of fashion market and management , 16, 216-233.

[3] De Neve, G. 2009. Power, inequality and corporate social responsibility: The politics of ethical compliance in the South Indian garment industry. Econ. Polit. Wkly. $44,63-72$.

[4] Dash, S.R.; Bhal, K.T.; Udgata, J. 2009. Organisational culture and environmental responsibility: A study of textile industry in India. International journmal of Indian Cutural Business Management , 2, 233-246.

[5] Baskaran, V.; Nachiappan, S.; Rahman, S. 2012. Indian textile suppliers' sustainability evaluation using the grey approach. International Journal of production Economics , 135, 647-658.

[6] Baskaran, V., Nachiappan, S., Rahman, S. 2011. Supplier assessment based on corporate social responsibility criteria in Indian automotive and textile industry sectors. International Journal of Sustainable Engineering , 4 359-369.

[7] Lauesen, L.M. 2011. CSR in publicly owned enterprises: Opportunities and barriers. Social Responsibility , 7, $558-577$.

[8] Arevalo, J.A. and Aravind, D. 2011. Corporate social responsibility practices in India: Approach, drivers, and barriers. Corporate Governanace , 11, 399-414.

[9] Faisal, M.N. 2010. Analysing the barriers to corporate social responsibility in supply chains: An interpretive structural modelling approach. Int. J. Logist. Res. Appl., 13, 179-195.

[10] Sweeney, L. 2007. Corporate social responsibility in Ireland: Barriers and opportunities experienced by SMEs when undertaking CSR. Corp. Gov., 7, 516-523.

[11] Garavan, T.N. , Heraty, N. , Rock, A. and Dalton, E.2010. Conceptualizing the behavioural barriers to CSR and CS in organizations: A typology of HRD interventions. Advance Development in Human resource , 12, 587-613.

[12] Laudal, T. 2011. Drivers and barriers of CSR and the size and internationalization of firms. Social Responsibility Journal , 7, 234-256.

[13] Govindan, K. , Kannan, D. and Shankar, K.M. 2014 Evaluating the drivers of corporate social responsibility in the mining industry with multi-criteria approach: A multi-stakeholder perspective. Journal of Cleaner production, doi:10.1016/j.jclepro.2013.12.065. 
[14] Perry, P. and Towers, N. 2009. Determining the antecedents for a strategy of corporate social responsibility by small-and medium-sized enterprises in the UK fashion apparel industry. Journal of retail consumer service , 16, 377-385.

[15] Chi, T. 2011. Building a sustainable supply chain: An analysis of corporate social responsibility (CSR) practices in the Chinese textile and apparel industry. J. Text. Inst. , 102, 837-848.

[16] Narayanan, A.E. Sridharan, R. Kumar, P.N.R. 2018. "Analyzing the interactions among barriers of sustainable supply chain management practices: A case study", Journal of Manufacturing Technology Management, https://doi.org/10.1108/JMTM-06-2017-0114

[17] Valmohammadi, C. 2011. Investigating corporate social responsibility practices in Iranian organizations: An ISO 26000 perspective. Bus. Strategy Ser. , 12, 257-263.

[18] Duarte, F.P. and Rahman, S. 2010. Perceptions of corporate social responsibility by Bangladeshi managers: An exploratory study. Int. Rev. Bus. Res. Pap., 6, 119136

[19] Mont, O. and Leire, C. 2009. Socially responsible purchasing in supply chains: Drivers and barriers in Sweden. Soc. Responsib. J., 5, 388-407.

[20] Pedersen, E.R.G. , Neergaard, P. , Pedersen, J.T. and Gwozdz, W. 2013. Conformance and deviance: Company responses to institutional pressures for corporate social responsibility reporting. Bus. Strategy Environ. , 22, 357-373.
[21] Goworek, H. 2011. Social and environmental sustainability in the clothing industry: A case study of a fair trade retailer. Soc. Responsib. J. , 7, 74-86.

[22] Kozar, J.M. and Connell, K.Y.H. 2013. Socially and environmentally responsible apparel consumption: Knowledge, attitudes, and behaviors. Soc. Responsib. J., 9, 315-324.

[23] Ha-Brookshire, J.E. and Norum, P.S. 2011. Willingness to pay for socially responsible products: Case of cotton apparel. J. Consum. Market., 28, 344-353.

[24] Dickson, M.A. and Eckman, M. 2006. Social responsibility: The concept as defined by apparel and textile scholars. Cloth. Text. Res. J., 24, 178-191.

[25] Cheung, Y.; Kong, D.; Tan, W.; Wang, W. 2015, Being good when being international in an emerging economy: The case of China. J. Bus. Ethics, 130, 805-817.

[26] Kronenberg, J. and Bergier, T. 2012. Sustainable development in a transition economy: Business case studies from Poland. J. Clean. Prod., 26, 18-27.

[27] Lewicka-Strzalecka, A. 2006. Opportunities and limitations of CSR in the post-communist countries: Polish case. Corp. Gov. Int. Rev. , 6, 440-448.

[28] Warfield, J.N. 1974. Developing interconnection matrices in structural modeling. IEEE Transactions on Systems, Man, and Cybernetics, (1), 81-87. 